\title{
P01.25. Classification of L6 muscle spindle afferents in the anesthesized cat
}

\author{
W Reed ${ }^{*}$, J Pickar \\ From International Research Congress on Integrative Medicine and Health 2012 \\ Portland, Oregon, USA. 15-18 May 2012
}

\section{Purpose}

Patients with low back pain demonstrate proprioceptive difficulties including spinal repositioning errors and impaired lumbosacral proprioceptive acuity. Few data are available regarding proprioceptive properties of muscle spindles in the low back. Muscle spindle afferents can originate from 2 types of receptive endings which terminate and respond to mechanical changes in 3 types of intrafusal fibers. Receptive endings may be primary or secondary. They terminate on dynamic bag $\left(b_{1}\right)$ static bag $\left(b_{2}\right)$ and/or chain $(c)$ intrafusal fibers. We sought to classify lumbar paraspinal muscle spindle afferents based upon their receptive endings and intrafusal terminations. Classification was based on their responses to ramp and hold vertebral movement before and after intrafusal activation using succinylcholine (SCH, 100-300ug/kg.ia). Afferents terminating in primary endings are especially responsive to the dynamic ramp stimulus. During intrafusal activation, afferents terminating on $b_{1}$ fibers further increase their discharge to the dynamic ramp whereas those terminating on $b_{2}$ fibers increase their static resting discharge.

\section{Methods}

Electrophysiological recordings from spindle afferents $(n=195)$ were obtained from $L_{6}$ dorsal root filaments with receptive fields in the $\mathrm{L}_{6}$ longissimus and multifidus muscles in an anesthetized cat preparation. Controlled vertebral actuations that stretched the paraspinal muscles were applied to the $\mathrm{L}_{6}$ spinous process in a dorsal-ventralward direction $[1.5 \mathrm{~mm} \quad(\mathrm{n}=120), 1.6 \mathrm{~mm}$ $(\mathrm{n}=21)$, or $1.7 \mathrm{~mm}(\mathrm{n}=54)$ using a feedback motor system. Instantaneous discharge frequency was averaged
Table 1

\begin{tabular}{lcccccc}
\hline & \multicolumn{3}{c}{ LONGISSIMUS } & \multicolumn{3}{c}{ MULTIFIDUS } \\
\hline & $\mathbf{b}_{\mathbf{1}} \mathbf{c}$ & $\mathbf{b}_{\mathbf{2}} \mathbf{c}$ & $\mathbf{b}_{\mathbf{1}} \mathbf{b}_{\mathbf{2}}$ & $\mathbf{b}_{\mathbf{1}} \mathbf{c}$ & $\mathbf{b}_{\mathbf{2}} \mathbf{c}$ & $\mathbf{b}_{\mathbf{1}} \mathbf{b}_{\mathbf{2}}$ \\
\hline Primary & 0 & 100 & 46 & 0 & 19 & 10 \\
\hline Secondary & 0 & 0 & 0 & 0 & 4 & 1 \\
\hline Intermediate & 0 & 5 & 0 & 0 & 1 & 0 \\
\hline
\end{tabular}

and compared over three ramp cycles pre- and post$\mathrm{SCH}$ injection.

\section{Results}

\section{Conclusion}

Almost all lumbar muscle spindle afferents showed static sensitivity responding as primary endings terminating on $b_{2}$ fibers. Approximately $1 / 3$ of the afferents responded as primary endings terminating on both $b_{1}$ and $b_{2}$ fibers. No endings were exclusively sensitive to the dynamic ramp stimulus.

Published: 12 June 2012

doi:10.1186/1472-6882-12-S1-P25

Cite this article as: Reed and Pickar: P01.25. Classification of L6 muscle spindle afferents in the anesthesized cat. BMC Complementary and Alternative Medicine 2012 12(Suppl 1):P25.

Palmer College of Chiropractic, Davenport, USA 